\title{
MicroRNA-455 regulates migration and invasion of human hepatocellular carcinoma by targeting Runx2
}

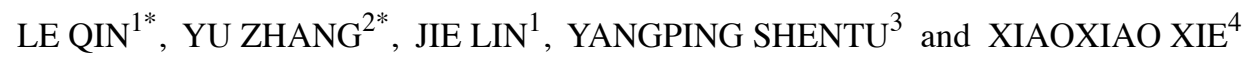 \\ ${ }^{1}$ Department of Pediatric Surgery, The Second Affiliated Hospital and Yuying Children's Hospital of \\ Wenzhou Medical University; ${ }^{2}$ Department of Hematology, The First Affiliated Hospital of \\ Wenzhou Medical University; ${ }^{3}$ Medical Function Center of Wenzhou Medical University; \\ ${ }^{4}$ Department of Medical Imaging, The Second Affiliated Hospital and Yuying Children's Hospital of \\ Wenzhou Medical University, Wenzhou, Zhejiang 325000, P.R. China
}

Received May 12, 2016; Accepted July 2, 2016

DOI: 10.3892/or.2016.5139

\begin{abstract}
MicroRNA-455 (miR-455) has been considered as a novel cancer-related miRNA and dysregulated expression frequently occurs in various human types of cancer. However, its clinical significance, its biological function and the underlying molecular signaling involved in hepatocellular carcinoma (HCC) remain to be elucidated. In the present study, we found that the expression level of miR-455 was significantly downregulated in both HCC tissues and cell lines. Low expression of miR-455 was significantly associated with poor prognostic features including multiple tumor nodes, high Edmondson-Steiner grading, advanced tumor-node-metastasis (TNM) stage and venous infiltration. In addition, our data revealed that miR-455 was a novel prognostic indicator for predicting the 5-year overall and disease-free survival of HCC patients. The gain- and loss-of-function studies revealed that miR-455 significantly suppressed migration and invasion of HCC cells in vitro. miR-455 was inversely correlated with runt-related transcription factor 2 (Runx2) expression in HCC samples. Moreover, we identified that miR-455 inversely regulated Runx2 expression in HCC cells. In this investigation, Runx2 was found to be a direct downstream target of miR-455. Evidently, alteration in Runx 2 expression suppressed the effect
\end{abstract}

Correspondence to: Professor Xiaoxiao Xie, Department of Medical Imaging, The Second Affiliated Hospital and Yuying Children's Hospital of Wenzhou Medical University, 109 Xueyuan West Road, Wenzhou, Zhejiang 325000, P.R. China

E-mail: xiexx1234@sina.com

Professor Yangping Shentu, Medical Function Center of Wenzhou Medical University, 82 Xueyuan West Road, Wenzhou, Zhejiang 325000, P.R. China

E-mail: q1_wenyi@sina.com

*Contributed equally

Key words: microRNA-455, hepatocellular carcinoma, runt-related transcription factor 2, migration, invasion of miR-455 on HCC cell migration and invasion. In conclusion, our data demonstrated that miR-455 promotes HCC growth by targeting Runx 2 and can potentially be regarded as a novel prognostic indicator and valuable therapeutic strategy for HCC.

\section{Introduction}

MicroRNAs (miRNAs) are a class of non-coding highly conserved RNAs that suppress protein expression through binding to the 3'-untranslated region (3'-UTR) of target mRNAs, and function as post-transcriptional regulators of gene expression (1). Increasing evidence has confirmed that miRNAs participate in diverse biological events such as differentiation, disease, cell proliferation, apoptosis, migration and invasion $(2,3)$. Previous studies revealed that miRNAs play important roles in human types of cancer and suggest that the aberrant behavior of miRNAs contribute to the development and progression of human types of cancer by modulating the expression of oncogenes or tumor suppressors (4-6).

miR-455 has been shown to be significantly dysregulated in different human tumors (7-11). For instance, it was markedly downregulated in basal cell, glioblastoma and colorectal carcinoma and in gastric and non-small cell lung cancer (12-17). It inhibited the proliferation, migration and invasion of lung cancer by directly modulating ZEB1 (18). Moreover, miR-455 inhibited proliferation and invasion of colorectal carcinoma cells by adjusting RAF proto-oncogene serine/threonine-protein kinase (RAF1) and could be a prognostic marker for colorectal cancer patients (19). Furthermore, miR-455 was a predictor of chemoresistance in colon adenocarcinoma and diffuse large B-cell lymphoma patients (20). These studies revealed that miR-455 serves as a suppressor miRNA in tumors. However, the functional role of miR-455 and the underlying signaling mechanism by which miR-455 regulates the development and progression of $\mathrm{HCC}$ have not been established.

The present research aimed to identify the biological function of miR-455 in HCC. We demonstrated that miR-455 was downregulated in HCC samples with an aggressive phenotype. Downregulation of miR-455 was found to be 
correlated with adverse prognostic characteristics and reduced 5-year overall and disease-free survival of HCC patients. Gainand loss-of-function experiments demonstrated that miR-455 inhibited HCC migration and invasion in vitro. Moreover, runt-related transcription factor 2 (Runx2) was confirmed as a direct target of miR-455. Our results elucidate the underlying mechanism involved through which miR-455 suppresses HCC and propose the use of miR-455 as a potential therapeutic strategy for HCC.

\section{Materials and methods}

Clinical specimens and data. One-hundred and four HCC specimens were obtained from patients undergoing curative resection of their primary HCC at the First and Second Affiliated Hospital of Wenzhou Medical University, from January 2006 to December 2008, with a median follow-up time of 40 months. The clinicopathological data were collected and are summarized in Table I. Informed consent was obtained and signed by all patients. Patients did not receive preoperative chemotherapy or embolization.

Cell line culture and treatment. The HCC cell lines (HepG2, Hep3B, SMMC-7721, MHCC-97H and Bel-7402) and the normal hepatocyte cell line L02 were purchased from the Institute of Biochemistry and Cell Biology (Chinese Academy of Sciences, Shanghai, China) and were maintained in Dulbecco's modified Eagle's medium (DMEM) supplemented with $10 \%$ fetal bovine serum (FBS; both from Gibco, Grand Island, NY, USA) in a $5 \% \mathrm{CO}_{2}$ humidified atmosphere at $37^{\circ} \mathrm{C}$.

The miRNA expression vectors, including the miR-455 expression vector (HmiR0148-MR03), the control vector (CmiR0001-MR04), miR-455 inhibitor (HmiR-AN0516) and the negative control (CmiR-AN0001-AM04) and Runx2 overexpression plasmid (LPP-H5215-lv101) were obtained from Genecopoeia (Guangzhou, China). The specific siRNA against Runx2, with sequence 5'-UAACAGCAGAGGCAUU UCGUAGCUC-3' and a scramble siRNA were produced by Sangon Biotech Co., Ltd. (Shanghai, China). Cells were transfected with the aforementioned vectors using Lipofectamine 2000 reagent (Invitrogen Life Technologies) in accordance with the manufacturer's instructions.

Quantitative real-time polymerase chain reaction ( $q R T$ $P C R)$. Total cellular RNA was isolated from the cultured cells or clinical tissues using TRIzol reagent (Invitrogen Life Technologies). RNA was reverse-transcribed using a TaqMan human miRNA assay kit (Applied Biosystems, Foster City, CA, USA) and cDNA was then amplified with a SYBR ${ }^{\circledR}$ Premix Ex Taq ${ }^{\mathrm{TM}}$ II (Perfect Real-Time) kit (Takara Bio Inc., Shiga, Japan). Hsa-miR-455 primer (HmiRQP0516), snRNA U6 qPCR Primer (HmiRQP9001), Runx2 (HQP016478) and GAPDH (HQP006940) were purchased from Genecopoeia.

Western blot analysis. Cultured cells and clinical tissues were collected and lysed and then the protein concentration was determined using a BCA Protein Assay kit (Thermo Fisher Scientific, Rockford, IL, USA). Thirty micrograms of denatured protein was subjected to $10 \%$ SDS-PAGE and transferred to a polyvinylidene fluoride (PVDF) membrane (Millipore,
Billerica, MA, USA). The membranes were incubated with respective primary antibodies: Runx 2 and GAPDH (1:1,000; Cell Signaling Technology, Inc.) at $4^{\circ} \mathrm{C}$ overnight. Then the membranes were washed three times with TBST and incubated with the appropriate HRP-conjugated secondary antibody for $2 \mathrm{~h}$ at room temperature (ZSGB-BIO Co., Ltd., Beijing, China). Protein bands were developed using an enhanced chemiluminescence kit (Amersham/GE Healthcare Life Sciences, Little Chalfont, UK).

Immunohistochemistry. Paraffin-embedded sections were subjected to immunohistochemical staining. Runx2 (1:300; Cell Signaling Technology, Inc.) antibody was applied as the primary antibody using a streptavidin peroxidase-conjugated (SP-IHC) method. The percentage of positive cells was expressed as: 0 for $<10 \%$; 1 for $10-30 \%$; 2 for $31-50 \%$; 3 for $>50 \%$.

Transwell assays. Transwell migration and invasion assays were carried out using $8-\mu \mathrm{m}$ pore-sized Transwell inserts (Nalge Nunc International Corp., Naperville, IL, USA). A cell suspension with a density of $2.5 \times 10^{5}$ cells $/ \mathrm{ml}$ in serumfree DMEM was prepared and $200 \mu \mathrm{l}$ of this cell suspension was loaded into the top chamber, and $750 \mu 1$ DMEM with $10 \%$ FBS was placed in the bottom chamber. After a 24-h incubation, cells were fixed in $4 \%$ paraformaldehyde for $15 \mathrm{~min}$ and stained with $0.3 \%$ crystal violet dye for $15 \mathrm{~min}$. The cells remaining in the top layer were swabbed carefully. The BioCoat Matrigel invasion chamber (Becton Dickinson Labware) was used to perform the Transwell invasion assay as previously described above.

Dual-luciferase reporter gene assay. The predicted Runx2 3'-UTR sequence and the corresponding mutated sequence of the sites were produced and embedded into the pRL-TK control vector (Promega, Corp., Madison, WI, USA). MHCC-97H cells which achieved $90 \%$ confluency were seeded in a 96-well plate. For the reporter assay, $120 \mathrm{ng}$ miR-455 inhibitor or negative control was co-transfected with $30 \mathrm{ng}$ of the wild-type (wt) or mutant (mt) 3'-UTR of Runx2 mRNA using $0.45 \mu \mathrm{l}$ of FuGene ${ }^{\circledR}$ (Promega) and then were transfected into the MHCC-97H cells. Following $48 \mathrm{~h}$ of transfection, the luciferase activity was measured according to the manufacturer's instructions (Dual-Luciferase assay system; Promega). The assays were repeated independently in triplicate.

Statistical analysis. Data are presented as the mean \pm SD and performed with at least three independent replicates. SPSS software, 16.0 (SPSS, Inc., Chicago, IL, USA) and GraphPad Prism 6.0 (GraphPad Software, Inc., La Jolla, CA, USA) were used with a two-tailed Student's t-test, Pearson's correlation analysis, the Kaplan-Meier method and the log-rank test to evaluate the statistical significance. Significant differences were defined as $\mathrm{P}<0.05$.

\section{Results}

miR-455 expression is downregulated in HCC tissues and cell lines. We investigated the expression of miR-455 compared to an endogenous control (U6 RNA) in 104 pairs 

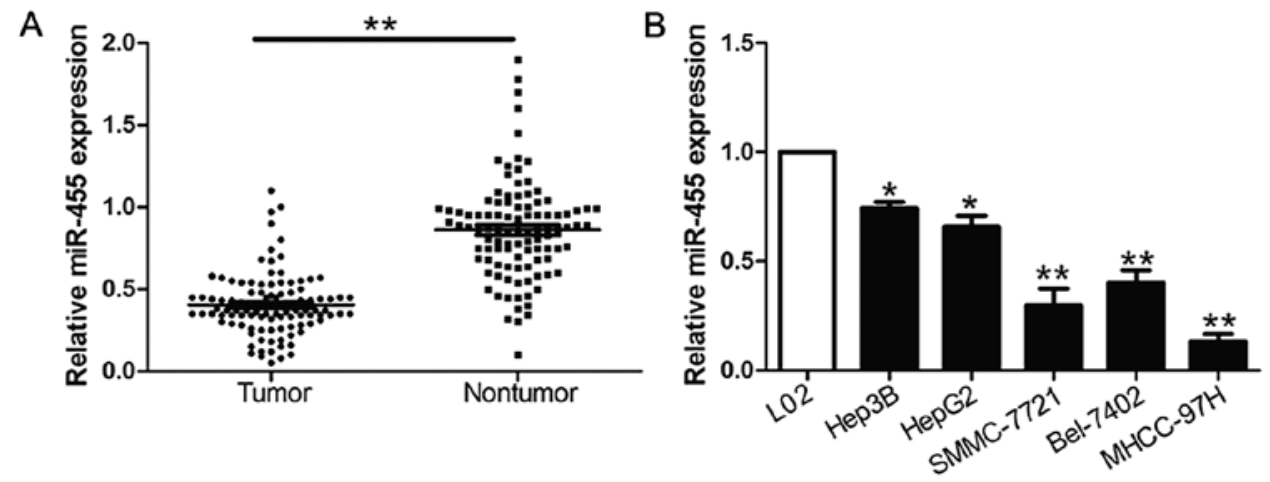

Figure 1. Expression of miR-455 in hepatocellular carcinoma (HCC) specimens and cell lines. (A) Quantification of the data revealed that the mean level of miR-455 expression in HCC tissues was significantly lower than that in the matched adjacent non-tumor tissues. (B) Differences in the expression levels of miR-455 between HCC cell lines compared to the immortalized hepatic cell line L02. Six repeats were performed with similar results. U6 snRNA was used as an internal control. ${ }^{*} \mathrm{P}<0.05,{ }^{* *} \mathrm{P}<0.01$.

Table I. Correlation between the clinicopathological characteristics and miR-455 expression in the HCC cases $(\mathrm{n}=104)$.

\begin{tabular}{|c|c|c|c|c|}
\hline \multirow[b]{2}{*}{$\begin{array}{l}\text { Clinical } \\
\text { parameters }\end{array}$} & \multirow[b]{2}{*}{$\begin{array}{c}\text { Cases } \\
\text { (n) }\end{array}$} & \multicolumn{2}{|c|}{ Expression level } & \multirow[b]{2}{*}{$\begin{array}{c}\text { P-value } \\
\left({ }^{a} \mathrm{P}<0.05\right)\end{array}$} \\
\hline & & $\begin{array}{c}\operatorname{miR}-455^{\text {high }} \\
\quad(\mathrm{n}=48)\end{array}$ & $\begin{array}{c}\mathrm{miR}-455^{\text {low }} \\
\quad(\mathrm{n}=56)\end{array}$ & \\
\hline Age (years) & & & & 0.451 \\
\hline$<65$ & 32 & 13 & 19 & \\
\hline$\geq 65$ & 72 & 35 & 37 & \\
\hline Gender & & & & 0.834 \\
\hline Male & 88 & 41 & 47 & \\
\hline Female & 16 & 7 & 9 & \\
\hline Tumor size $(\mathrm{cm})$ & & & & 0.716 \\
\hline$<5$ & 50 & 24 & 26 & \\
\hline$\geq 5$ & 54 & 24 & 30 & \\
\hline Tumor no. & & & & $0.025^{\mathrm{a}}$ \\
\hline Solitary & 86 & 44 & 42 & \\
\hline Multiple & 18 & 4 & 14 & \\
\hline Edmondson & & & & $0.001^{\mathrm{a}}$ \\
\hline $\mathrm{I}+\mathrm{II}$ & 35 & 24 & 11 & \\
\hline III+IV & 69 & 24 & 45 & \\
\hline TNM stage & & & & $0.008^{\mathrm{a}}$ \\
\hline $\mathrm{I}+\mathrm{II}$ & 81 & 43 & 38 & \\
\hline III+IV & 23 & 5 & 18 & \\
\hline Venous & & & & \\
\hline infiltration & & & & $0.021^{\mathrm{a}}$ \\
\hline Present & 21 & 5 & 16 & \\
\hline Absent & 83 & 43 & 40 & \\
\hline $\operatorname{AFP}(\mathrm{ng} / \mathrm{ml})$ & & & & 0.544 \\
\hline$<400$ & 29 & 12 & 17 & \\
\hline$\geq 400$ & 75 & 36 & 39 & \\
\hline HBsAg & & & & 0.966 \\
\hline Positive & 89 & 41 & 48 & \\
\hline Negative & 15 & 7 & 8 & \\
\hline
\end{tabular}

aStatistically significant. HCC, hepatocellular carcinoma; TNM, tumor-node-metastasis; AFP, $\alpha$-fetoprotein; HBsAg, hepatitis B surface antigen. of HCC tissues and the corresponding non-tumor tissues. We found that miR-455 expression in HCC tissues was significantly lower than levels noted in the corresponding adjacent non-tumor tissues $(\mathrm{P}<0.05$, Fig. 1A). Furthermore, we analyzed miR-455 expression in a non-transformed hepatic cell line (L02) and five HCC cell lines (HepG2, Hep3B, SMCC-7721, Bel-7402 and MHCC-97H). Notably, our results revealed that the relative expression of miR- 455 was evidently downregulated in the HCC cell lines when compared with the L02 cells $(\mathrm{P}<0.05$, Fig. 1B). These data suggest that the downregulated expression of miR-455 potentially correlates with the development and progression of HCC.

Clinical significance of the downregulation of miR-455 expression in HCC samples. We defined two different miR-455 expression groups according to the mean expression level of miR-455 in order to investigate the association between miR-455 expression levels and the clinical characteristics and prognosis of the HCC patients. As shown in Table I, low expression of miR-455 was clearly correlated with multiple tumor nodes $(\mathrm{P}=0.025)$, venous infiltration $(\mathrm{P}=0.021)$, high Edmondson-Steiner grading $(\mathrm{P}=0.001)$ and advanced tumornode-metastasis (TNM) tumor stage $(\mathrm{P}=0.008)$. Hence, these findings revealed that the reduced expression of miR-455 was correlated with adverse prognostic characteristics of HCC. Furthermore, Kaplan-Meier analysis revealed that high expression of miR-455 was significantly correlated with a prolonged overall survival $(\mathrm{P}=0.0001$, Fig. 2A) and disease-free survival of the HCC patients $(\mathrm{P}=0.0001$, Fig. 2B). Moreover, miR-455 expression was found to be a novel independent factor for predicting both the 5-year overall and disease-free survival in HCC patients $(\mathrm{P}=0.018$ and 0.011, respectively; Table II). Our results suggest that miR-455 could serve as a valuable biomarker for predicting the outcome of HCC patients.

miR-455 suppresses HCC cell migration and invasion. To identify the biological function of miR-455 in HCC, we transduced HCC cell lines with the miR-455 expression vector or anti-miR-455 vector with different endogenous miR-455 levels. As determined by qRT-PCR, the miR-455 vector obviously increased the expression level of miR-455 in the 

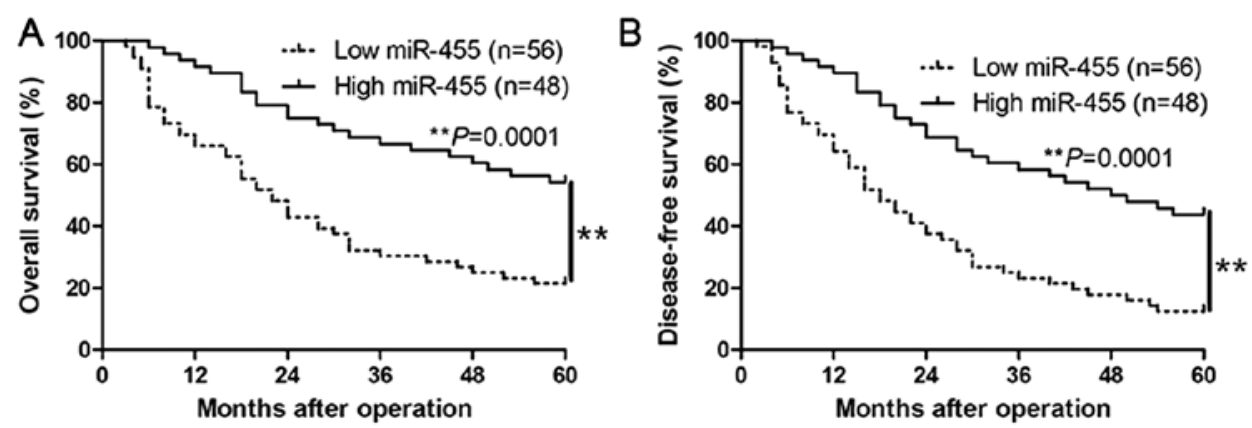

Figure 2. Prognostic value of miR-455 for hepatocellular carcinoma (HCC) patients. HCC patients with higher expression of miR-455 had better (A) overall survival and (B) disease-free survival. ${ }^{* *} \mathrm{P}<0.01$.

Table II. Multivariate Cox regression analysis of the 5-year overall and disease-free survival of the 104 HCC patients.

\begin{tabular}{|c|c|c|c|c|c|c|}
\hline \multirow[b]{2}{*}{ Variables } & \multicolumn{3}{|c|}{ Overall survival } & \multicolumn{3}{|c|}{ Disease-free survival } \\
\hline & HR & $95 \% \mathrm{CI}$ & $\mathrm{P}$-value & HR & $95 \% \mathrm{CI}$ & P-value \\
\hline miR-455 expression & 2.179 & $1.113-4.249$ & $0.018^{\mathrm{a}}$ & 3.602 & $1.113-4.794$ & $0.011^{\mathrm{a}}$ \\
\hline Edmondson grade & 1.213 & $0.713-1.983$ & 0.230 & 1.123 & $0.694-1.869$ & 0.347 \\
\hline TNM stage & 2.812 & $1.355-5.813$ & $0.007^{\mathrm{a}}$ & 2.194 & $1.020-4.649$ & $0.009^{\mathrm{a}}$ \\
\hline No. of tumor nodules & 1.694 & $0.894-3.024$ & 0.068 & 1.674 & $0.999-2.815$ & 0.056 \\
\hline Venous infiltration & 3.253 & $2.108-5.983$ & $0.001^{\mathrm{a}}$ & 3.521 & $2.238-6.372$ & $0.001^{\mathrm{a}}$ \\
\hline
\end{tabular}

aStatistically significant. HCC, hepatocellular carcinoma; HR, hazard ratio; CI, confidence interval; TNM, tumor-node-metastasis .

MHCC-97H cells ( $\mathrm{P}<0.01$, Fig. 3A), while the anti-miR-455 vector significantly reduced the expression level of miR-455 in the Hep3B cells $(\mathrm{P}<0.01$, Fig. 3C). Transwell migration and invasion assays showed that increased expression of miR-455 in the MHCC-97H cells (MHCC-97H-miR-455) resulted in an obvious decrease in cell migration and invasion $(\mathrm{P}<0.05$, Fig. $3 \mathrm{~B})$, while downregulation of miR-455 in Hep3B cells (Hep3B-anti-miR-455) showed a marked increase in the number of migrated and invaded cells $(\mathrm{P}<0.05$, Fig. 3D). Collectively, miR-455 may function as an anti-metastatic molecule in HCC.

Runx2 is a direct target of miR-455 in HCC. To further confirm the mechanism involved in the miR-455 regulation in HCC cells, we explored the candidate target genes of miR- 455 by searching the publically available databases TargetScan 6.2 and miRanda. As shown in Fig. 4A, we demonstrated that 3 '-UTR of Runx2 contained the highly conserved complementary sequence of miR-455. Moreover, Zhang et al reported that Runx 2 is a target of miR-455 in chondrogenic differentiation (13). To confirm this prospect, we first investigated the relationship between miR-455 and Runx2 in the HCC cases. Our data revealed that both the Runx 2 mRNA and protein levels in the miR-455 highexpressing tumor tissues were obviously lower than those in the miR-455 low-expressing tumors $(\mathrm{P}<0.05$, Fig. $4 \mathrm{~B}$ and $\mathrm{C})$. In addition, the expression level of miR-455 was inversely associated with the expression level of Runx 2 mRNA in the HCC tissues ( $r=0.669, \mathrm{P}<0.0001$, Fig. 4D). Similarily, as determined by the IHC results, the protein expression level of Runx 2 was significantly decreased in patients with a high level of miR-455 ( $\mathrm{P}<0.05$, Fig. 4E). Furthermore, the expression levels of the Runx 2 mRNA and protein were prominently inhibited by the overexpression of miR-455 in the MHCC-97H cells $(\mathrm{P}<0.05$, respectively, Fig. 5A and B). By contrast, the downregulation of miR-455 prominently promoted the mRNA and protein expression levels of Runx2 in the Hep3B cells $(\mathrm{P}<0.05$, respectively, Fig. $5 \mathrm{~A}$ and $\mathrm{B})$. In addition, the overexpression of miR-455 clearly suppressed the luciferase activity of Runx 2 containing a wt 3'-UTR but did not inhibit the activity of $m t$ Runx2 ( $<<0.001$, Fig. 5C). Suppression of miR-455 increased the luciferase activity of wt Runx2-3'-UTR ( $\mathrm{P}<0.001$, Fig. 5C). However, with the mt Runx2-3'-UTR vectors, there was no relative increase in activity. Collectively, these data strongly suggest that Runx 2 is a downstream target of miR-455 in HCC.

Alteration of Runx2 expression levels affect the functional effects of miR-455 in HCC cells. To explore that Runx 2 is a functional target of miR-455, we restored the Runx2 expression in MHCC-97H-miR-455 cells by transfecting them with a Runx2 plasmid ( $\mathrm{P}<0.05$, Fig. $6 \mathrm{~A})$. We found that restoration of Runx 2 expression in the MHCC-97H-miR-455 cells partially suppressed the effect of exogenous miR-455, resulting in a prominent increase in cell migration and invasion $(\mathrm{P}<0.05$, Fig. 6B). Similarly, silencing of Runx2 in Hep3B-anti-miR-455 cells 

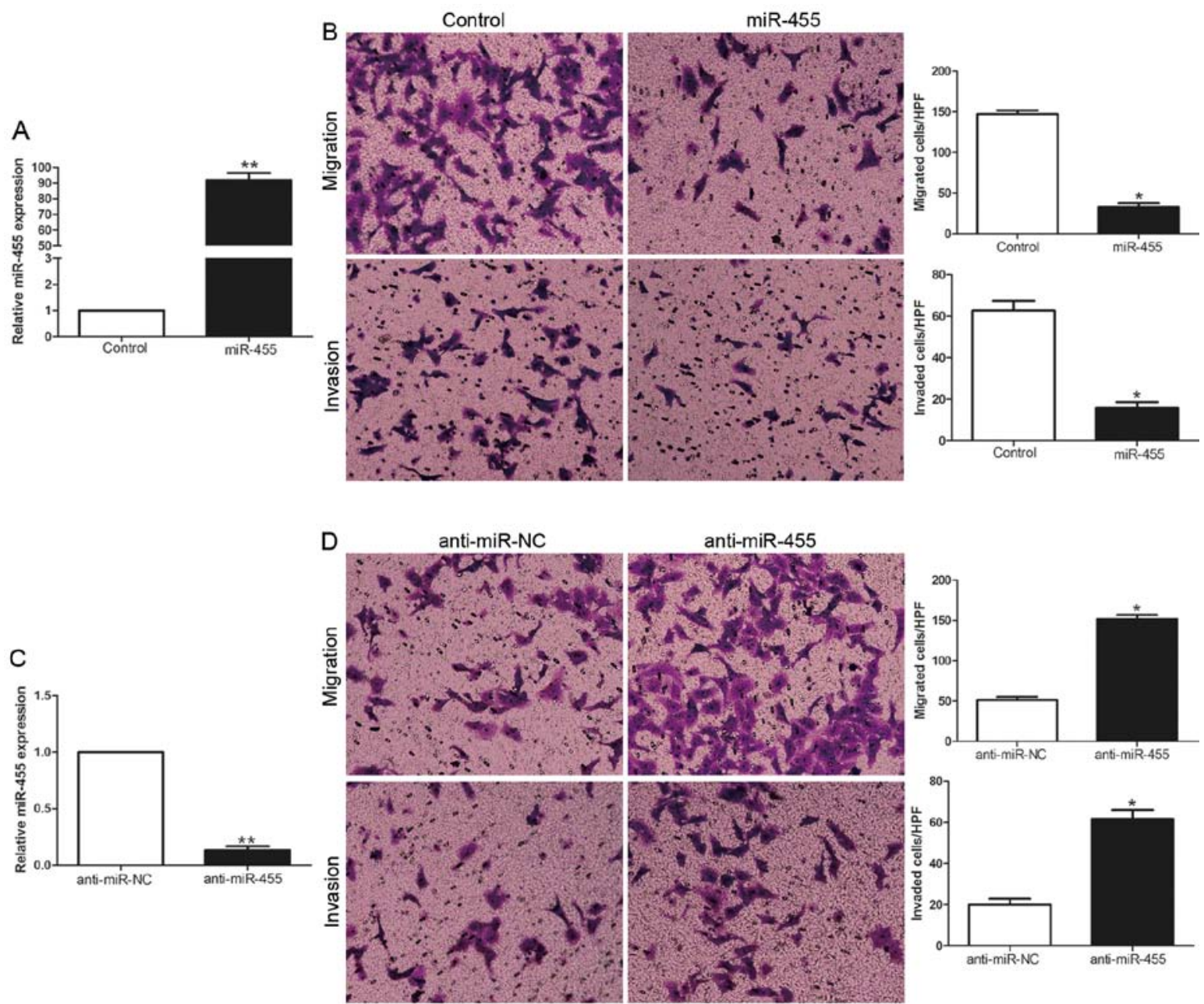

Figure 3. miR-455 inhibits hepatocellular carcinoma (HCC) cell migration and invasion. (A) MHCC-97H cells that were transfected with corresponding miRNA vectors were subjected to RT-qPCR for miR-455. (B) Cell migration and invasion as measured by Transwell assays were inhibited by overexpression of miR-455 in MHCC-97H cells. (C) Hep3B cells that were transfected with miR-455 inhibitors (anti-miR-455) and negative control were subjected to qRT-PCR for miR-455. (D) Cell migration and invasion as measured by Transwell assays were increased by knockdown of miR-455 in Hep3B cells. Six independent experiments were performed. ${ }^{*} \mathrm{P}<0.05,{ }^{* * *} \mathrm{P}<0.01$.

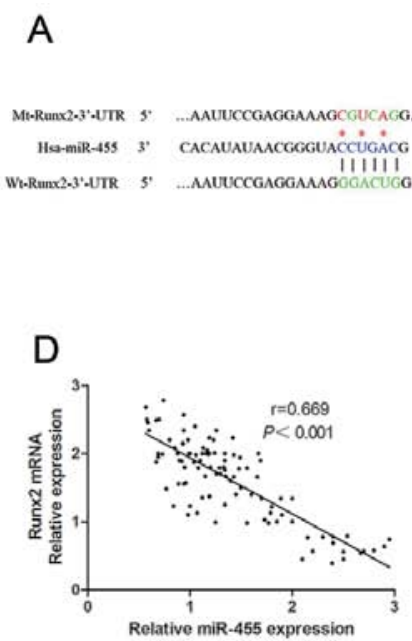

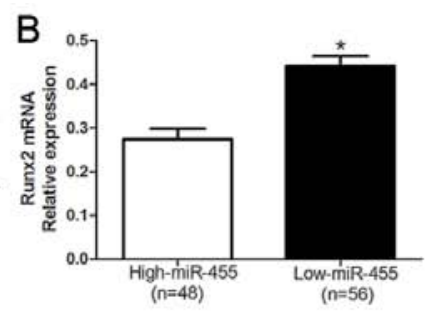

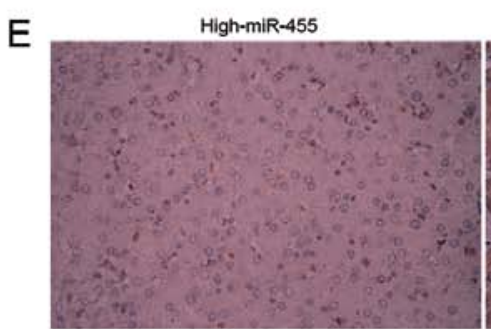

C
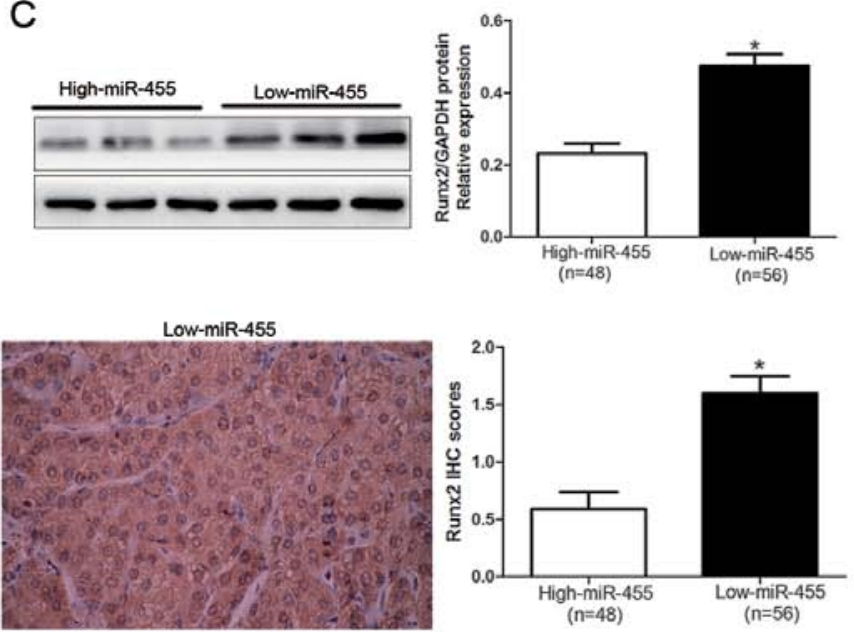

Figure 4. Inverse correlation between miR-455 and runt-related transcription factor 2 (Runx2) expression levels observed in hepatocellular carcinoma (HCC), (A) miR-455 and its putative binding sequence in the 3'-untranslated region (3'-UTR) of Runx2. The mutant binding site was generated in the complementary site for the seed region of miR-455. (B) The expression of Runx 2 mRNA in the miR-455 high-expressing tumors was significantly lower than that in the miR-455 low-expressing tumors. (C) The expression of Runx2 protein in the miR-455 high-expressing tumors was significantly lower than that in the miR-455 low-expressing tumors. (D) A significant inverse correlation between the mRNA expression levels of Runx2 and miR- 455 was observed in HCC tissues. (E) Representative immunohistochemical staining showed a weak staining of Runx2 in the miR-455 high-expressing HCC tissues and a strong staining of Runx2 in the miR-455 low-expressing tumors. " $\mathrm{P}<0.05$. 

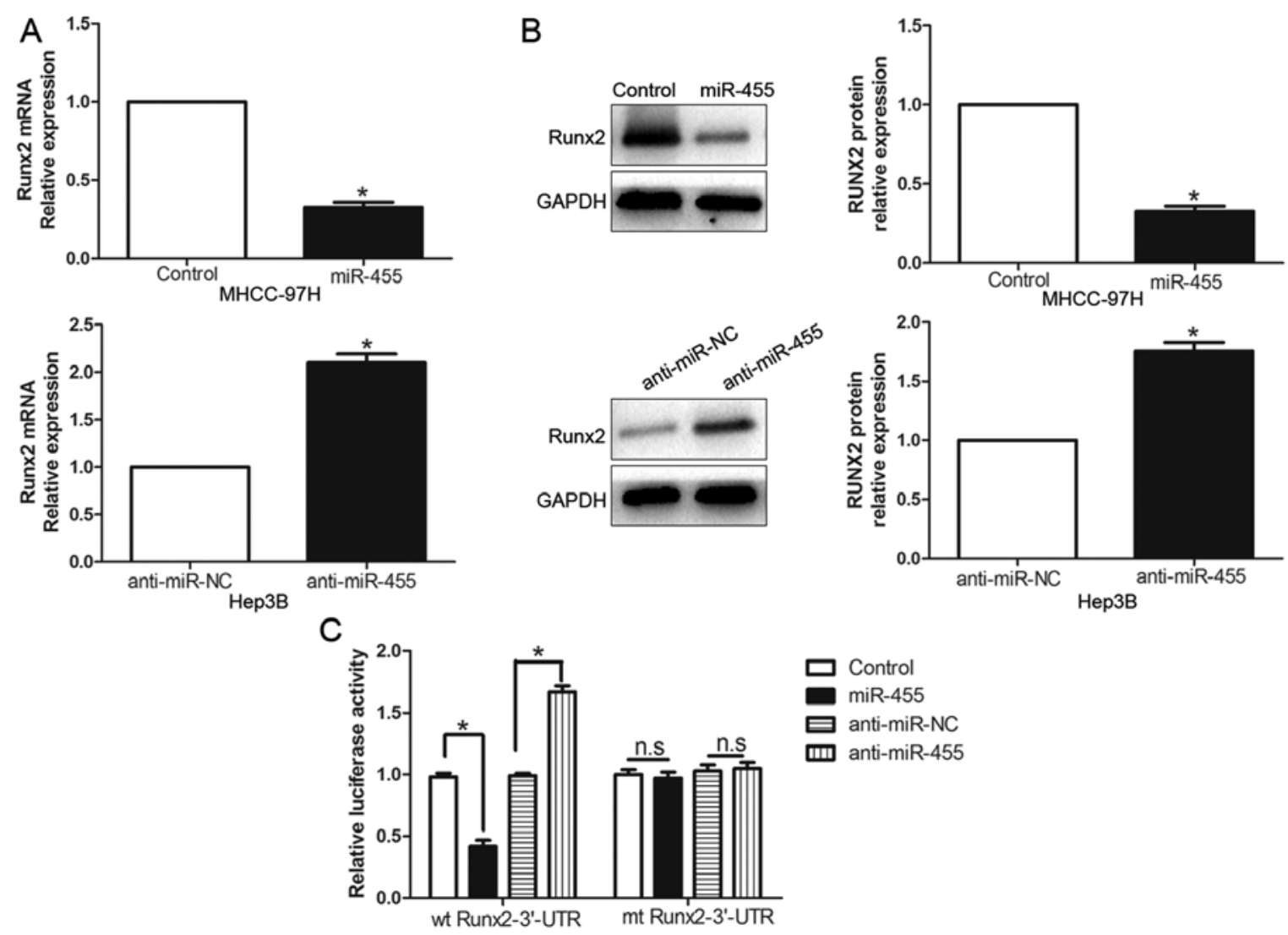

Figure 5. Runt-related transcription factor 2 (Runx2) identified as a direct target of miR-455 in hepatocellular carcinoma (HCC). (A) RT-qPCR analysis of Runx2 mRNA expression in MHCC-97H cells with miR-455 or miR-control transfection vector and Hep3B cells with anti-miR-455 or anti-miR-NC transfection vector. (B) Overexpression of miR-455 reduced the expression of the Runx2 protein in MHCC-97H cells and knockdown of miR-455 increased the expression level of the Runx2 protein in Hep3B cells. (C) miR-455 significantly suppressed the luciferase activity that carried the wild-type (wt) but not the mutant (mt) 3'-untranslated region (3'-UTR) of Runx2. Anti-miR-455 led to a notable increase in the luciferase activity of wt 3'-UTR of Runx2. Six repeats were performed with similar results, ${ }^{*} \mathrm{P}<0.05$; n.s., not significant.

partially suppressed the effect of anti-miR-455 on migration and invasion $(\mathrm{P}<0.05$, Fig. $6 \mathrm{C}$ and $\mathrm{D})$. These data demonstrate that Runx 2 is a downstream mediator in the function of miR-455 in HCC.

\section{Discussion}

Increasing research has reported the critical roles of miRNAs in the initiation and progression of various human types of cancer (5). Exploration of cancer-specific miRNAs and their functional molecular mechanism contributes to the identification of novel biomarkers and therapeutic strategies for human types of cancer (6). In this study, we identified that the mean expression level of miR-455 was obviously decreased in HCC tissues compared with that observed in the corresponding tumor-adjacent tissues. Consistently, we also observed that miR-455 was decreased in HCC cell lines vs. a normal hepatic cell line. These data suggest that miR-455 may be a novel tumor suppressor in HCC. Furthermore, low expression of miR-455 was significantly correlated with multiple tumor nodes, venous infiltration, high Edmondson-Steiner grading and advanced TNM tumor stage. These data indicate that the downregulated expression of miR-455 is associated with adverse prognostic characteristics in HCC. Moreover, we confirmed that increased expression of miR-455 predicted a significant better 5-year survival for HCC patients. Multivariate Cox repression analysis demonstrated that miR- 455 is a novel independent prognostic indicator for predicting the survival of HCC patients. Collectively, these data reveal that miR-455 is critical for the prognostic outcome in HCC patients.

Previous studies have confirmed miR-455 as a suppressor in human types of cancer. Functionally, miR-455 exerts anticancer functions by inhibiting tumor growth and metastasis (21). Moreover, miR-455 suppressed epithelial-mesenchymal transition (EMT) in NSCLC by targeting ZEB1 (18). These results were consistent with the observations we performed in our experiments. In the present study, we demonstrated that miR-455 overexpression clearly reduced the number of migrated and invaded MHCC-97H cells and miR-455 knockdown significantly promoted Hep3B cell migration and invasion. Furthermore, we identified that miR-455 suppressed migration and invasion, at least in part, by targeting Runx2. miR-455 was inversely associated with the expression levels of both Runx 2 mRNA and protein in HCC tissues. miR-455 negatively regulated Runx2 accumulation in HCC cells. Moreover, the complementary sequence of miR-455 was confirmed in the 3'-UTR of Runx2 mRNA. Knockdown of miR-455 increased the luciferase reporter activity of wt 3'-UTR but not mt 3'-UTR of Runx2. Conversely, the overexpression of miR-455 inhibited the 
A
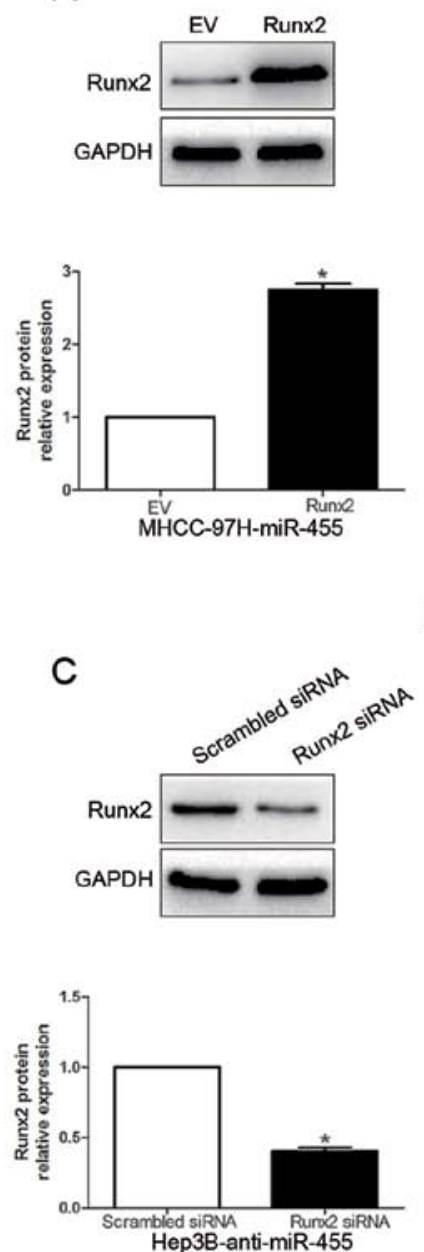

B

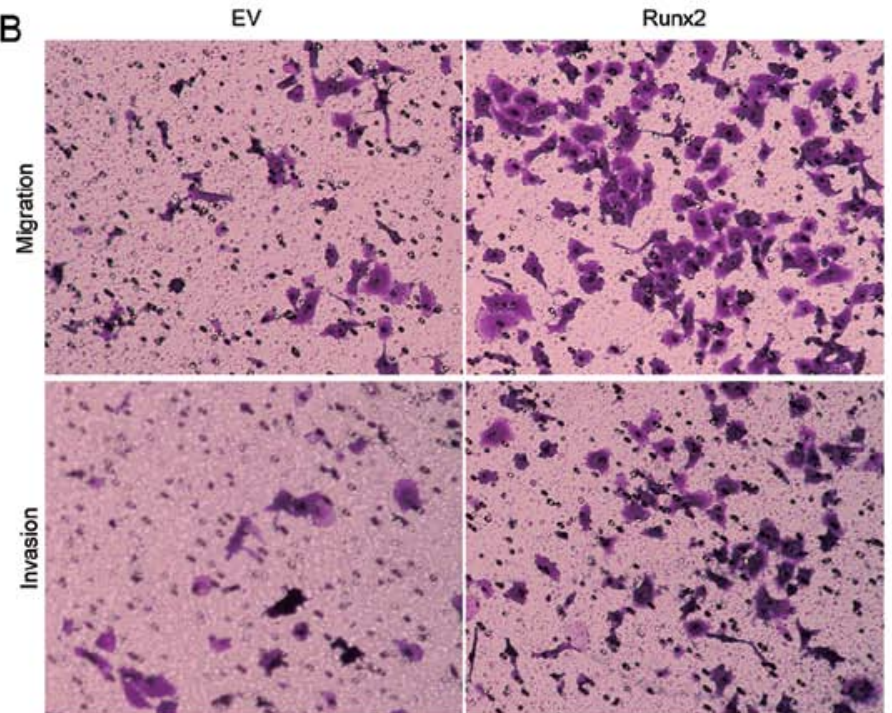

D

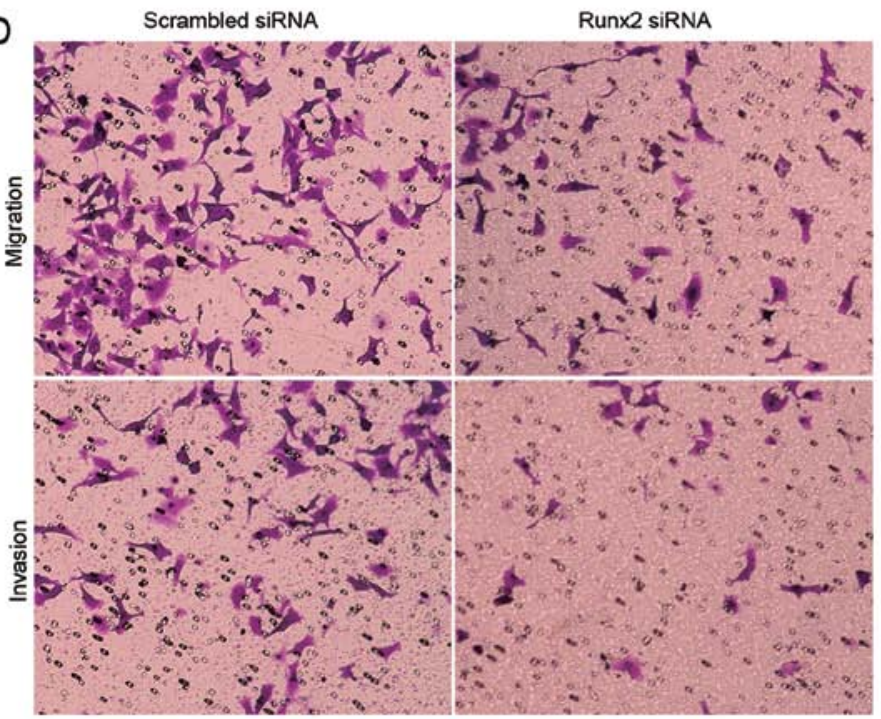

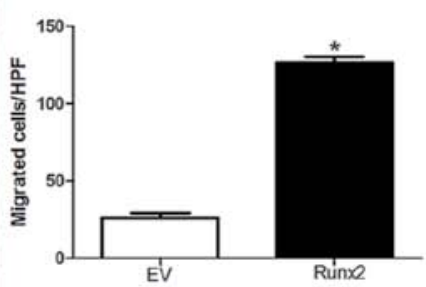

MHCC-97H-miR-455
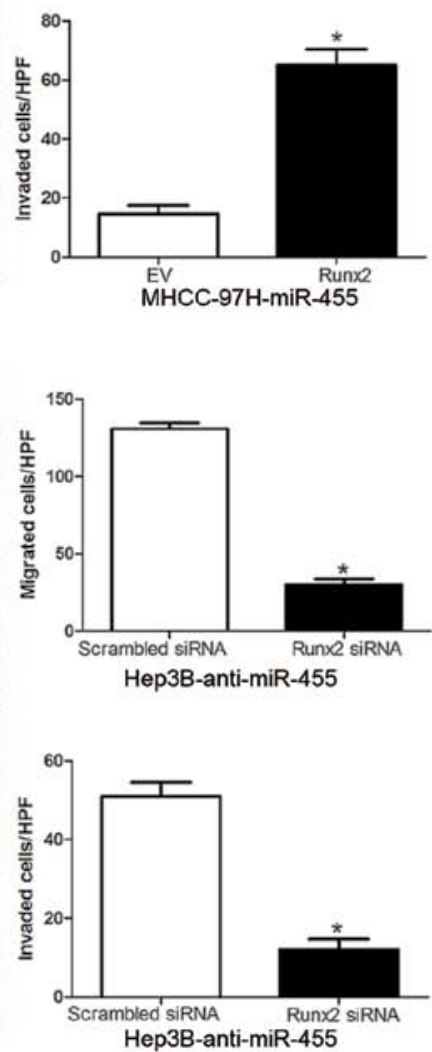

Figure 6. Alterations of runt-related transcription factor 2 (Runx2) partially suppress miR-455-mediated hepatocellular carcinoma (HCC) cell migration and invasion. (A) miR-455-overexpressing MHCC-97H cells that were transfected with an empty vector (EV) or a Runx2 plasmid were subjected to western blot analysis for Runx2. (B) Cell migration and invasion of the miR-455-overexpressing MHCC-97H cells were increased by Runx2 overexpression. (C) miR-455-knockdown Hep3B cells that were transfected with scrambled siRNA or Runx2 siRNA were subjected to western blot analysis for Runx2.

(D) Runx 2 knockdown abrogated the effects of miR-455 knockdown in HCC cells. Six independent experiments were performed. "P<0.05.

luciferase activity of wt 3'-UTR but not mt 3'-UTR of Runx2. The function of miR-455 alteration on migration and invasion of HCC cells was also dissipated by Runx 2 modulation. In addition, Runx 2 has been reported to be a direct downstream target of miR-455 in early chondrogenic differentiation (13). Collectively, our results support Runx2 as a downstream mediator of miR-455 function in HCC.

Previous studies have demonstrated that Runx 2 regulates tumor progression, invasion and metastasis and its function in migration and invasion has been documented in different tumor cell types (22-26). Furthermore, Runx 2 overexpression upregulated transcription factors (SOX9, SNAI2 and SMAD3) that participate in the process of EMT (27-29) and whose features include increased motility and invasion potential. Moreover, Runx 2 was found to directly regulate the expression levels of MMP9 and MMP13 and mediate the invasion of human breast cancer cell lines (30). It has been shown that Runx 2 plays a critical role in hepatocarcinogenesis. This mechanism may account for the effects of miR-455 in $\mathrm{HCC}$.

In conclusion, the data revealed that the expression of miR-455 was downregulated in HCC tissues and cell lines and its low expression is related to malignant clinicopathological characteristics. We confirmed that miR-455 is an independent prognostic marker for predicting the 5-year survival of HCC patients. In vitro, studies demonstrated that miR-455 suppressed HCC cell migration and invasion. Mechanistically, its tumor-suppressive effects are mediated by Runx2. Collectively, the downregulation of miR-455 may play a critical role in tumor migration and invasion and may be a novel prognostic factor and of potential use in therapeutic strategies for HCC. 


\section{References}

1. Mendell JT: MicroRNAs: Critical regulators of development, cellular physiology and malignancy. Cell Cycle 4 $1179-1184,2005$

2. Bartel DP: MicroRNAs: Genomics, biogenesis, mechanism, and function. Cell 116: 281-297, 2004.

3. Szabo G and Bala S: MicroRNAs in liver disease. Nat Rev Gastroenterol Hepatol 10: 542-552, 2013.

4. Lu J, Getz G, Miska EA, Alvarez-Saavedra E, Lamb J, Peck D, Sweet-Cordero A, Ebert BL, Mak RH, Ferrando AA, et al: MicroRNA expression profiles classify human cancers. Nature 435: 834-838, 2005.

5. Calin GA and Croce CM: MicroRNA signatures in human cancers. Nat Rev Cancer 6: 857-866, 2006.

6. Jansson MD and Lund AH: MicroRNA and cancer. Mol Oncol 6: 590-610, 2012

7. Liu C, Iqbal J, Teruya-Feldstein J, Shen Y, Dabrowska MJ, Dybkaer K, Lim MS, Piva R, Barreca A, Pellegrino E, et al: MicroRNA expression profiling identifies molecular signatures associated with anaplastic large cell lymphoma. Blood 122 2083-2092, 2013.

8. Hummel R, Wang T, Watson DI, Michael MZ, Van der Hoek M, Haier J and Hussey DJ: Chemotherapy-induced modification of microRNA expression in esophageal cancer. Oncol Rep 26: 1011-1017, 2011.

9. Pathak S, Meng WJ, Nandy SK, Ping J, Bisgin A, Helmfors L, Waldmann P and Sun XF: Radiation and SN38 treatments modulate the expression of microRNAs, cytokines and chemokines in colon cancer cells in a p53-directed manner. Oncotarget 6: 44758-44780, 2015.

10. Shoshan E, Mobley AK, Braeuer RR, Kamiya T, Huang L, Vasquez ME, Salameh A, Lee HJ, Kim SJ, Ivan C, et al: Reduced adenosine-to-inosine miR-455-5p editing promotes melanoma growth and metastasis. Nat Cell Biol 17: 311-321, 2015.

11. Hudson J, Duncavage E, Tamburrino A, Salerno P, Xi L, Raffeld M, Moley J and Chernock RD: Overexpression of miR-10a and miR-375 and downregulation of YAP1 in medullary thyroid carcinoma. Exp Mol Pathol 95: 62-67, 2013.

12. Sand M, Skrygan M, Sand D, Georgas D, Hahn SA, Gambichler T, Altmeyer P and Bechara FG: Expression of microRNAs in basal cell carcinoma. Br J Dermatol 167: 847-855, 2012.

13. Zhang Z, Hou C, Meng F, Zhao X, Zhang Z, Huang G, Chen W, Fu M and Liao W: MiR-455-3p regulates early chondrogenic differentiation via inhibiting Runx2. FEBS Lett 589: 3671-3678, 2015.

14. Boisen MK, Dehlendorff C, Linnemann D, Nielsen BS, Larsen JS, Osterlind K, Nielsen SE, Tarpgaard LS, Qvortrup C, Pfeiffer $\mathrm{P}$, et al: Tissue microRNAs as predictors of outcome in patients with metastatic colorectal cancer treated with first line capecitabine and oxaliplatin with or without bevacizumab. PLoS One 9: e109430, 2014.

15. Bera A, VenkataSubbaRao K, Manoharan MS, Hill P and Freeman JW: A miRNA signature of chemoresistant mesenchymal phenotype identifies novel molecular targets associated with advanced pancreatic cancer. PLoS One 9: e106343, 2014.

16. Hamilton MP, Rajapakshe K, Hartig SM, Reva B, McLellan MD, Kandoth C, Ding L, Zack TI, Gunaratne PH, Wheeler DA, et al: Identification of a pan-cancer oncogenic microRNA superfamily anchored by a central core seed motif. Nat Commun 4 2730,2013
17. Hiroki E, Akahira J, Suzuki F, Nagase S, Ito K, Suzuki T, Sasano $\mathrm{H}$ and Yaegashi N: Changes in microRNA expression levels correlate with clinicopathological features and prognoses in endometrial serous adenocarcinomas. Cancer Sci 101: 241-249, 2010.

18. Li YJ, Ping C, Tang J and Zhang W: MicroRNA-455 suppresses non-small cell lung cancer through targeting ZEB1. Cell Biol Int 40: 621-628, 2016.

19. Chai J, Wang S, Han D, Dong W, Xie C and Guo H: MicroRNA-455 inhibits proliferation and invasion of colorectal cancer by targeting RAF proto-oncogene serine/threonine-protein kinase. Tumour Biol 36: 1313-1321, 2015.

20. Song G, Gu L, Li J, Tang Z, Liu H, Chen B, Sun X, He B, Pan Y, Wang S, et al: Serum microRNA expression profiling predict response to R-CHOP treatment in diffuse large B cell lymphoma patients. Ann Hematol 93: 1735-1743, 2014

21. Yang $\mathrm{H}$ and Wang Y: Five miRNAs considered as molecular targets for predicting neuroglioma. Tumour Biol 37: 1051-1059, 2015.

22. Baniwal SK, Khalid O, Gabet Y, Shah RR, Purcell DJ, Mav D, Kohn-Gabet AE, Shi Y, Coetzee GA and Frenkel B: Runx2 transcriptome of prostate cancer cells: Insights into invasiveness and bone metastasis. Mol Cancer 9: 258, 2010.

23. Akech J, Wixted JJ, Bedard K, van der Deen M, Hussain S, Guise TA, van Wijnen AJ, Stein JL, Languino LR, Altieri DC, et al: Runx2 association with progression of prostate cancer in patients: Mechanisms mediating bone osteolysis and osteoblastic metastatic lesions. Oncogene 29: 811-821, 2010.

24. Brusgard JL, Choe M, Chumsri S, Renoud K, MacKerell AD Jr, Sudol $M$ and Passaniti A: RUNX2 and TAZ-dependent signaling pathways regulate soluble E-cadherin levels and tumorsphere formation in breast cancer cells. Oncotarget 6: 28132-28150, 2015.

25. Tandon M, Chen Z and Pratap J: Runx2 activates PI3K/Akt signaling via mTORC2 regulation in invasive breast cancer cells. Breast Cancer Res 16: R16, 2014.

26. van der Deen M, Akech J, Wang T, FitzGerald TJ, Altieri DC, Languino LR, Lian JB, van Wijnen AJ, Stein JL and Stein GS: The cancer-related Runx 2 protein enhances cell growth and responses to androgen and TGF $\beta$ in prostate cancer cells. J Cell Biochem 109: 828-837, 2010.

27. Cohen-Solal KA, Boregowda RK and Lasfar A: RUNX2 and the PI3K/AKT axis reciprocal activation as a driving force for tumor progression. Mol Cancer 14: 137, 2015.

28. Niu DF, Kondo T, Nakazawa T, Oishi N, Kawasaki $T$, Mochizuki K, Yamane T and Katoh R: Transcription factor Runx2 is a regulator of epithelial-mesenchymal transition and invasion in thyroid carcinomas. Lab Invest 92: 1181-1190, 2012.

29. Tandon M, Chen Z, Othman AH and Pratap J: Role of Runx2 in IGF-1R $\beta / A$ kt- and AMPK/Erk-dependent growth, survival and sensitivity towards metformin in breast cancer bone metastasis. Oncogene 35: 4730-4740, 2016.

30. Wysokinski D, Blasiak J and Pawlowska E: Role of RUNX2 in breast carcinogenesis. Int J Mol Sci 16: 20969-20993, 2015. 